\title{
Diagnóstico del consumo del calamar gigante en México y en Sonora
}

\author{
Ma. Consepción Luna Raya* \\ José Isabel Urciaga García*** \\ César Augusto Salinas Zavala* \\ Miguel Ángel Cisneros Mata*** \\ Luis Felipe Beltrán Morales*
}

\begin{abstract}
The aim of this paper is to diagnose the consumption of giant squid in Mexico and Sonora. We have carried out a comparative analysis of the nutritional properties and the consumption levels between giant squid and meat products. In order to describe the aspects related to the taste and preferences of the consumer of giant squid, we have used results from 1,799 questionnaires applied nationally, in 14 cities. The results of the poll indicate that the consumption of giant squid is low in terms of frequency and quantity. However, we have found a trend towards the consumption of giant squid with added value. In general terms, there are opportunities to encourage the consumption of giant squid both nationally and regionally.
\end{abstract}

Keywords: giant squid, consumption, nutritional characteristics, taste, preferences.

\section{Resumen}

El objetivo del trabajo es presentar un diagnóstico del consumo del calamar gigante en México y Sonora. Se realizó un análisis comparativo de las propiedades nutricionales y de los niveles de consumo del calamar gigante y los productos cárnicos. Para describir los aspectos relacionados con gustos y preferencias del consumidor del calamar gigante, se utilizaron resultados de 1,799 encuestas aplicadas en 14 ciudades del país. Los resultados de las encuestas indican que el consumo del calamar gigante, en frecuencia y cantidad, es bajo; no obstante, se encontró una tendencia hacia el consumo del calamar gigante con valor agregado. En términos generales, existen oportunidades para fomentar el consumo del calamar gigante en los ámbitos nacional y regional.

Palabras clave: calamar gigante, consumo, características nutricionales, gustos y preferencias.

* Centro de Investigaciones Biológicas del Noroeste. Correos-e: mluna04@cibnor. mx, csalinas@cibnor.mxy lbeltran04@cibnor.mx.

** Universidad Autónoma de Baja California Sur. Correo-e: jurciaga@uabcs.mx.

*** Programa Golfo de California del Fondo Mundial para la Naturaleza. Correoe: macisne@yahoo.com. 


\section{Introducción}

En la estructura y desarrollo del sector pesquero del Golfo de California, en el Pacífico mexicano, una de las pesquerías que más ha llamado la atención es la del calamar gigante (Dosidicus gigas; D’Orbigny, 1835). Ello se explica por la creciente importancia en los volúmenes de captura total de esa pesquería en la última década, representando una fuente significativa de empleos e ingresos en la región, particularmente en Sonora. El impulso de esa pesquería está fuertemente asociado con la demanda externa, particularmente con la evolución de los mercados asiáticos, mercados con un patrón de consumo que incluye cantidades importantes de calamar gigante. Así, la dinámica de las capturas y su importancia económica están gobernadas por la dinámica de las exportaciones mexicanas a los mercados asiáticos, principalmente el coreano. En general, el producto se exporta como materia prima con poco valor agregado y a bajo precio. Cesár A. Salinas y otros autores (2004: 6) sugieren que pese a la falta de costumbre de considerarlo en la dieta de los mexicanos, actualmente existe potencial en el mercado nacional para fomentar el consumo del calamar gigante, producto que paulatinamente se empieza a introducir como un sustituto de otras especies marinas, entre las que destaca el pulpo. Al parecer, el bajo consumo del calamar gigante en México y en Sonora obedece al desconocimiento de sus características nutricionales y de las formas de preparación.

El objetivo de este trabajo es presentar un diagnóstico del consumo del calamar gigante en el mercado nacional y en Sonora. En el documento se exponen antecedentes de la pesquería del calamar gigante en el noroeste de México, se destacan las principales características nutricionales de ese producto, y se compara el mismo con el grupo de alimentos cárnicos. Se utiliza el consumo nacional aparente y per cápita como un indicador comparativo entre el grupo de alimentos pesqueros y el de cárnicos. A partir del análisis de gustos y preferencias de los consumidores, se hace una descripción de la situación del consumo del calamar gigante en el país (año 2004). Finalmente, se hacen algunas sugerencias para fomentar el consumo de productos pesqueros en general, y en especial del calamar gigante, en el mercado nacional. 


\section{Antecedentes}

El calamar gigante, Dosidicus gigas (D’Orbigny, 1835), es un molusco marino cefalópodo que pertenece a la familia Ommastrephidae. De las especies de calamar, el Dosidicus gigas se explota en forma comercial en México y su captura se registra de manera oficial; la captura se realiza en el Golfo de California, y se descarga en los puertos de Mazatlán, Sinaloa; Santa Rosalía, Baja California Sur, y Guaymas, Sonora. El atractivo comercial del calamar gigante radica en su gran abundancia y en la calidad y contenido nutritivo de su carne. Sin embargo, el consumo nacional de calamar es bajo debido a que ha sido poco difundido, a pesar de las características mencionadas y de su bajo precio ( $\mathrm{Sa}-$ linas et al., 2004: 6).

La abundancia de calamar en el Golfo de California es variable, y ello se refleja en la disponibilidad para la flota pesquera. La captura del calamar gigante en el litoral del Pacífico mexicano en la segunda mitad de la década de los noventa aumentó en 1,900\%, al pasar de 6,226 toneladas en 1994 a 120,877 en 1997. En el año de 1998, la captura de calamar fue de 26,611 toneladas, lo que representó una disminución respecto al año anterior. Esta variación negativa en la captura fue asociada con el fenómeno "El Niño". ${ }^{1}$ Sin embargo, de 1999 al 2002 la captura se incrementó en 100\%, al pasar de 57,985 toneladas en 1999 a 115,896 en la temporada de 2002 (Conapesca, 2000, 2002, 2003).

La captura comercial del calamar gigante dentro del Golfo de California ha sido muy importante para la economía de la región. Ha tenido efectos positivos dentro de la industria pesquera, manifestándose en la generación de empleos, tanto en la etapa de captura como en la de procesamiento.

\footnotetext{
${ }^{1}$ Cuando se presenta el fenómeno "El Niño", la señal se propaga a lo largo de las costas de América, llegando en algunas ocasiones a afectar el Golfo de California. Esta afectación se refiere a que al calentarse la masa de agua superficial, la termoclina se hunde, produciendo con ello un empobrecimiento de la masa oceánica superficial, afectando así toda la cadena trófica. Al escasear el alimento, el calamar gigante se desplaza a áreas donde sí encuentra alimento, en este caso a Bahía Magdalena, en la costa occidental de Baja California. El fenómeno "El Niño" que aquí se comenta terminó a finales de 1998, recuperándose las condiciones de alta productividad dentro del Golfo de California en el año 1999, lo cual, se presume, es la razón por la cual el calamar gigante se estableció nuevamente en el golfo, originando buenas capturas a partir de ese año.
} 
La etapa de comercialización ${ }^{2}$ del calamar gigante ha mostrado un comportamiento estable en los últimos años. En el mercado nacional, el calamar gigante se comercializa principalmente en la forma de manto o filete fresco-congelado y fresco-enhielado, tentáculos o "bailarina" (cabeza con tentáculos) y aleta. Recientemente, en el mercado nacional se está comercializando el calamar gigante enlatado, principalmente en la presentación de calamar en su tinta y calamar imitación abulón. La producción del calamar gigante enlatado se inició de manera experimental con la elaboración de productos como calamar en salmuera y crema de calamar (Klett, 1996: 148). Es importante señalar que las rutas de comercialización del calamar gigante en el mercado nacional son complejas de entender dada la dinámica del propio mercado en términos de negociaciones entre comercializadores mayoristas y minoristas (Salinas et al., 2004: 17).

\section{Características nutricionales y precios de los productos pesqueros y cárnicos}

\subsection{Características nutricionales de los productos pesqueros y cárnicos}

Con objeto de establecer puntos de referencia, se ubicó dentro del grupo de productos pesqueros al calamar gigante y al pulpo, ${ }^{3}$ y dentro del grupo de los productos cárnicos, a la carne de pollo, res y cerdo. En México, la ingesta de proteínas de origen animal está representada, en orden de importancia, por el consumo de pollo, res y cerdo, y de productos de origen marino en general. Pero el calamar gigante y el pulpo representan una parte mínima dentro la canasta de alimentos de la población nacional.

\footnotetext{
${ }^{2}$ Dentro de todo proceso productivo, la fase de la comercialización, entendida como la etapa de la distribución que comienza con la compra al productor de sus mercancías y concluye con su venta al consumidor final, juega un papel determinante en el comportamiento de dicho proceso, impactando en forma trascendental la fijación del precio del producto y el consumo del mismo (Fernández, 1986: 347). El proceso de comercialización representa la etapa necesaria de mediación para que el ciclo económico se complete y puedan ser realizados los productos en el mercado. Dentro del sector pesquero se presenta un permanente desequilibrio en la comercialización de algunos productos, debido fundamentalmente a la inconsistencia de los mercados existentes tanto en el ámbito nacional como en el internacional (Moctezuma, 1989: 145).

${ }^{3}$ Para efectos comparativos se consideró al pulpo por ser un producto similar al calamar gigante en apariencia y sabor, características que son apreciadas por los consumidores de estos moluscos.
} 
Los productos cárnicos representan una fuente importante de calorías y de proteínas ${ }^{4}$ para el consumidor nacional. El total de las calorías consumidas en México proviene en $83.5 \%$ de los productos de origen vegetal y en $16.5 \%$ de los de origen animal; y la aportación al total de calorías de los principales tipos de carne es de la siguiente forma: la carne de cerdo, 3.1\%; la de aves, $1.9 \%$, y la de bovino y de ternera, $1.7 \%$. El consumo total de proteínas deriva en $60.5 \%$ de productos de origen vegetal y en $39.5 \%$ de productos de origen animal; y dentro del consumo total de proteínas de origen animal, la participación es como sigue: carne de bovino y ternera, 7.8\%; de aves, 5.3\%, y de cerdo, 3.7\% (Martínez, 2001: 13-17).

Dentro del grupo de alimentos de origen marino, el calamar gigante destaca por la calidad de su carne. Es un producto con alto nivel nutritivo del que puede aprovecharse hasta $75 \%$ de sus partes después de quitársele las vísceras (Salinas et al., 2003: 42). Una de las razones de la importancia del aprovechamiento del calamar gigante, y en general de los moluscos, es su alto valor nutritivo, ya que contienen vitaminas A, B y D, compuestos glicerofosfóricos, cloruros, carbohidratos y proteínas en cantidades adecuadas y de fácil digestión. Las proteínas que están presentes son digeribles casi en su totalidad (Cifuentes et al., 1997: cap. 3), contra una digestibilidad de las proteínas de carnes en general de $94 \pm 3 \%$ (FAO-OMS-ONU, 1985).

El calamar gigante es un producto de importantes características nutricionales. Tiene un bajo contenido de grasas y un elevado valor nutritivo, que se atribuyen principalmente a sus componentes químicos, entre los que destacan las proteínas y el colágeno, que pueden ser variables en función de la edad del calamar gigante, estadio de madurez, e incluso sitio y temporada de captura (Ezquerra et al., 2002). La carne del calamar gigante es de una consistencia suave y posee un delicado pero característico sabor (Klett, 1996: 142).

En todos los mercados, la tendencia al consumo de productos “sanos" es creciente. En el caso del calamar gigante, en lo que se

${ }^{4}$ En las tablas internacionales sobre recomendaciones de necesidad energética para una dieta sana en un individuo sano, según la edad y sexo de la persona, se sugiere una ingesta promedio para menores de 18 años de 2,014 kcal/día/persona para hombres, y de 1,746 para mujeres; para personas mayores de 18 años se recomienda 2,388 kcal/día, para hombres y mujeres. En cuanto a las proteínas se recomienda una ingesta promedio, para menores de 18 años, de 28 gramos/día para hombres y de 27 gramos/día para mujeres; para mayores de 18 años se recomienda, para hombres, 45 gramos/día, y 38 gramos/día para mujeres (ONU/CEPAL, 1991). 
refiere a los aspectos nutricionales se puede señalar que la relación taurina/colesterol se ha convertido en un elemento clave para ampliar el mercado de ese producto. La taurina es un aminoácido compuesto que suprime la acumulación de colesterol en el cuerpo, y, por ende, la concentración de colesterol en la sangre casi nunca se eleva. La taurina tiene otras funciones: reduce la grasa neutra, mantiene la presión arterial y previene la diabetes al proporcionar la secreción de insulina. Si bien es cierto que el cuerpo sintetiza taurina, su ingestión directa vía alimentos que la contengan es recomendable (Klett, 1996: 148). Además del consumo de productos sanos, se ha observado una tendencia creciente al consumo de productos con valor agregado y de fácil preparación. ${ }^{5}$

De acuerdo con los datos presentados en el cuadro 1, el calamar gigante aporta, entre otros elementos, una cantidad de proteínas ${ }^{6}$ similar a la de los productos cárnicos de consumo generalizado como el pollo, la res y el cerdo. Contiene, además, una baja cantidad de calorías y un bajo contenido de grasas y de ácidos grasos saturados ${ }^{7}$ en comparación con los productos cárnicos presentados en el mismo cuadro.

\subsection{Precios de los productos pesqueros y cárnicos}

En esta sección se presentan los precios al consumidor del calamar y del pulpo, así como de la carne de res, cerdo y pollo. En el cuadro 2 se presentan los precios al consumidor reportados por la Procuraduría Federal del Consumidor (Profeco) durante la primera semana de noviembre de 2004 en los centros comerciales de la Ciudad de México, que es el mercado más representativo y en donde existe una gran concentración de todo tipo de productos.

\footnotetext{
${ }^{5}$ Aurora Zugarramurdi y María A. Parín (1998) mencionan que en el futuro próximo, una gran proporción de las capturas de pescados y mariscos se cambiará del área de bienes intermedios ("commodities") hacia productos con mayor valor agregado. Esa tendencia se incrementará con la creciente urbanización en los países desarrollados y en vías de desarrollo. Con ello se crean oportunidades de negocios para aquellos que conozcan el mercado y estén en condiciones de utilizar la tecnología y administración apropiadas.

${ }^{6}$ Es importante destacar que las proteínas del calamar gigante son de alta calidad y poseen los 10 aminoácidos esenciales para el cuerpo humano (Córdova, 2005).

${ }^{7}$ Las grasas saturadas se encuentran en los productos cárnicos en proporciones importantes, pero en los pescados y mariscos son casi nulas; estas grasas no son esenciales y elevan el colesterol. Los aceites poliinsaturados (como el omega 3 y el omega 6) son esenciales y se encuentran principalmente en los pescados y mariscos (Comunicación personal, licenciado en Nutrición Erick Legoff Engwall, Secretaría de Desarrollo Rural, Gobierno de Jalisco).
} 


\section{Cuadro 1}

Características nutricionales de productos cárnicos y pesqueros (datos por cada 100 gramos de porción comestible)

\begin{tabular}{|c|c|c|c|c|c|}
\hline Producto & & & & & \\
\hline Componente & Salamar (1) & Pulpo (2) & Pollo (3) & $\operatorname{Res}(4)$ & Cerdo (5) \\
\hline Agua (g) & 81.0 & 84.8 & 68.6 & 71.6 & 47.8 \\
\hline Proteínas (g) & 16.4 & 12.6 & 20.2 & 20.4 & 13.4 \\
\hline Grasas (g) & 1.1 & 1.0 & 11.1 & 6.3 & 37.8 \\
\hline Cenizas (g) & 1.5 & 1.6 & 1.4 & 0.7 & \\
\hline $\begin{array}{l}\text { Carbohidratos } \\
\text { totales }(\mathrm{g})\end{array}$ & 0.0 & 0.0 & & 0.5 & \\
\hline $\begin{array}{l}\text { Carbohidratos } \\
\text { disponibles (g) }\end{array}$ & 0.0 & 0.0 & & 0.5 & \\
\hline Energía (kcal) & 76 & 59 & 167 & 142 & 180 \\
\hline $\begin{array}{l}\text { Ácidos grasos } \\
\text { saturados (g) }\end{array}$ & 0.3 & & 3.2 & 2.5 & 13.8 \\
\hline $\begin{array}{l}\text { Ácidos grasos } \\
\text { monoinsaturados }\end{array}$ & ss (g) 0.2 & & 0.6 & & 16.2 \\
\hline $\begin{array}{l}\text { Ácidos grasos } \\
\text { poliinsaturados (g }\end{array}$ & (g) 0.5 & & 2.1 & & 3.6 \\
\hline Colesterol (mg) & & & 67 & 62 & 74 \\
\hline Sodio (mg) & & 89 & 65 & 63 & 44 \\
\hline Potasio (mg) & & 274 & 204 & 358 & 244 \\
\hline Calcio (mg) & 12 & 39 & 11 & 6.0 & 5.0 \\
\hline Fósforo (mg) & 119 & 109 & 196 & 179 & \\
\hline Hierro (mg) & 0.5 & 2.5 & 0.8 & 2.3 & 0.7 \\
\hline Zinc (mg) & 4.0 & 1.7 & 0.9 & 4.4 & 1.6 \\
\hline $\begin{array}{l}\text { Vitamina A Equiv. } \\
\text { totales }(\mu \mathrm{g})\end{array}$ & & & 39 & 6.0 & 2.0 \\
\hline Tiamina (mg) & 0.02 & 0.02 & 0.06 & 0.11 & 0.57 \\
\hline Riboflavina (mg) & 0.12 & 0.07 & 0.09 & 0.19 & 0.21 \\
\hline Niacina $(\mathrm{mg})$ & & 1.3 & 8.9 & 3.6 & $3.9(1)$ \\
\hline
\end{tabular}

(1) Calamar gigante fresco.

(2) Pulpo fresco.

(3) Pollo fresco (pechuga con piel).

(4) Carne magra de res (con muy poca grasa y sin hueso).

(5) Carne de cerdo en canal.

Notas: Los datos de la tabla corresponden a productos cuyo país de origen es México. Se podrá apreciar que existen en la tabla algunos valores faltantes, principalmente de micronutrientes (vitaminas y minerales), pues de acuerdo con la última actualización (octubre de 2002) no se disponía de dicha información.

Fuente: FAO-Latinfoods (2002). 


\section{Cuadro 2 \\ Precio de productos cárnicos (res, cerdo, pollo) y pesqueros (calamar, pulpo)}

\begin{tabular}{lccc}
\hline Producto & \multicolumn{3}{c}{ Precio (pesos / kilo a granel) } \\
\cline { 2 - 4 } & Mínimo & Máximo & Promedio \\
\hline Calamar en filete & 11.50 & 13.97 & 12.74 \\
Calamar en tentáculo & 12.40 & 17.90 & 15.15 \\
Pulpo entero & 50.90 & 60.00 & 55.45 \\
Bistec de res & 51.80 & 64.32 & 58.06 \\
Carne molida de res & 36.57 & 57.82 & 47.20 \\
Chuleta de cerdo & 44.73 & 66.90 & 55.82 \\
Milanesa de cerdo & 42.88 & 54.00 & 48.44 \\
Pierna de pollo & 22.21 & 26.90 & 24.56 \\
Pollo entero & 17.50 & 19.91 & 18.71 \\
\hline
\end{tabular}

Fuente: Profeco (2004).

El cuadro comparativo demuestra que el bajo precio del calamar gigante representa para el consumidor una ventaja en términos económicos. Esta característica ubica al calamar como una alternativa de consumo respecto a otros productos, tanto cárnicos como pesqueros. Por ejemplo, el calamar puede ser considerado como un sustituto del pulpo, ya que presenta características en sabor y en apariencia similares; además, el calamar registra precios promedio comparativamente menores a los del pulpo (cuadro 2).

\section{Estructura del consumo de productos pesqueros y cárnicos}

\subsection{Comparación de los niveles de consumo de los productos pesqueros y cárnicos}

La ingesta de proteínas de origen animal en México está representada, en orden de importancia, por el consumo de pollo, res y cerdo (Sagarpa, 2002). En comparación con los productos cárnicos, la demanda de productos pesqueros es menor a pesar de su valor nutritivo, lo cual puede estar originado por factores tales como el poder de compra, ${ }^{8}$ los gustos y preferencias, ${ }^{9}$ la falta de

${ }^{8}$ Por ejemplo, respecto al gasto en alimento que se realiza en los hogares, Patricia Moctezuma y otros autores (1989: 174) mencionan que el consumo de alimento de origen marino en Baja California sin duda está determinado por el nivel de ingresos de la población, sus gustos y preferencias; estos últimos motivados por los hábitos alimenticios desprendidos de culturas, tradiciones y creencias.

${ }^{9}$ Las preferencias de los consumidores hacen referencia a las razones por las que las personas prefieren un bien u otro con objeto de maximizar su bienestar (Pindyck y Rubinfeld, 2001: 64-76). 
costumbre, y la falta de promoción para el consumo de productos de origen marino. Moisés Valencia (1988: 79) señala que tratándose de alimentos como pescados y mariscos, la demanda depende no sólo del precio y del comportamiento del producto en el mercado; es decir, si su demanda aumenta o disminuye conforme aumenta el precio, sino que también depende del sabor, la textura, la presentación del producto, además de otras preferencias del consumidor.

El consumo nacional aparente ${ }^{10}$ y per cápita ${ }^{11}$ de productos pesqueros y cárnicos en México ha tenido variaciones importantes, tal como se muestra en los cuadros 3 y 4 . El consumo nacional aparente de productos pesqueros tuvo una disminución de 1.1\% de 1997 a 2002; sin embargo, en el caso del consumo del calamar gigante se registró un aumento de 14.9\%. La disminución en el consumo aparente del calamar gigante registrada en el año 1998 podría ser explicada por la caída en los niveles de captura, dada la disminución en la abundancia del recurso. Esa disminución tuvo su origen en las variaciones climáticas y de temperatura del agua asociadas con el evento de "El Niño", que provocaron también cambios en la estructura de tallas de los organismos, por lo que predominaron organismos de 24 centímetros de longitud de manto (Rivera, 2001), cuando en el Golfo de California se han capturado organismos de 70 a 83 centímetros de longitud de manto (Klett, 1996; Markaida, 2001).

Por otro lado, en lo referente a los productos cárnicos el consumo nacional aparente de pollo aumentó 40.4\% de 1997 a 2002; en el mismo periodo, el consumo de res registró un aumento de 19.1\%, y el de carne de cerdo aumentó 34.5\%.

El consumo per cápita de productos pesqueros en México registró un comportamiento variable durante el mismo periodo. En términos generales, se puede decir que existe un bajo consumo per cápita de productos pesqueros, pues éste ha presentado una tendencia decreciente en los últimos años, pasando de 13.0 kilogramos en 1997 a 11.5 en 2002. En 1998, el consumo per cápita fue de 10.7 kilogramos, lo cual, como ya se indicó, puede estar relacionado con las consecuencias del fenómeno "El Niño". El consumo per cápita del calamar gigante presentó una tendencia

${ }^{10} \mathrm{El}$ consumo nacional aparente de productos pesqueros es un indicador que determina el volumen de producto, en peso de desembarque, que se orienta al mercado interno para consumo humano directo e indirecto. Resulta de sumar las importaciones a la producción nacional, restándole las exportaciones (Conapesca, 2002).

${ }^{11}$ El consumo per cápita es un indicador que resulta de dividir el consumo nacional aparente entre la población total (Conapesca, 2002). 


\begin{tabular}{|c|c|c|c|c|c|}
\hline Const & laciona & $\begin{array}{r}\mathrm{Cl} \\
\text { (to }\end{array}$ & $\begin{array}{l}\text { adro } 3 \\
\text { e product } \\
\text { eladas) }\end{array}$ & pesqu & y cárnicos \\
\hline Año & $\begin{array}{l}\text { Productos } \\
\text { pesqueros }\end{array}$ & Calamar & Pollo & Res & Cerdo \\
\hline 1997 & $1 ’ 227,919$ & 64,155 & $1^{\prime} 609,482$ & 1'470,793 & 1'112,418 \\
\hline 1998 & 1’031,493 & 17,248 & 1’799,864 & 1'570,676 & $1 ’ 218,152$ \\
\hline 1999 & 1’109,820 & 25,377 & 1’931,332 & $1^{\prime} 582,893$ & $1 ’ 270,487$ \\
\hline 2000 & $1 ’ 215,599$ & 34,286 & $2^{\prime} 054,533$ & $1^{\prime} 622,993$ & $1 ’ 361,656$ \\
\hline 2001 & $1 ’ 238,636$ & 44,994 & $2 ’ 170,989$ & $1^{\prime} 642,995$ & $1 ' 503,385$ \\
\hline 2002 & $1 ’ 214,361$ & 73,726 & $2 ’ 259,450$ & $1 ’ 752,341$ & $1 ’ 495,896$ \\
\hline
\end{tabular}

Fuente: Para pollo, res y cerdo de 1997-2001, Sagarpa, Coordinación General de Ganadería (2002). Para pollo, res y cerdo de 2002, Presidencia de la Republica, Poder Ejecutivo Federal (2003). Para productos pesqueros y calamar, Conapesca $(2001,2002)$.

\section{Cuadro 4}

Consumo nacional per cápita de productos cárnicos y pesqueros (kilogramos)

\begin{tabular}{cccccc}
\hline Año & $\begin{array}{c}\text { Productos } \\
\text { pesqueros }\end{array}$ & Calamar & Pollo & Res & Cerdo \\
\hline 1997 & 13.0 & 0.7 & 17.0 & 15.5 & 12.0 \\
1998 & 10.7 & 0.2 & 18.7 & 16.3 & 12.7 \\
1999 & 11.2 & 0.3 & 20.0 & 16.4 & 13.1 \\
2000 & 12.5 & 0.4 & 20.6 & 16.3 & 13.7 \\
2001 & 12.2 & 0.4 & 21.8 & 16.5 & 15.1 \\
2002 & 11.5 & 0.7 & n.d. & n.d. & n.d. \\
\hline
\end{tabular}

Fuente: Para pollo, res y cerdo de 1997-2001, Sagarpa, Coordinación General de Ganadería (2002). Para pollo, res y cerdo de 2002, Presidencia de la Republica, Poder Ejecutivo Federal (2003). Para productos pesqueros y calamar, Conapesca $(2001,2002)$.

similar a la descrita anteriormente. Este indicador registró un máximo de 0.7 kilogramos, tanto en 1997 como en 2002, presentando un mínimo nivel de consumo en 1998 (0.2 kg), debido a la disminución en la oferta de este producto en el mercado nacional por los factores ambientales mencionados anteriormente, que limitaron las capturas del calamar gigante en el Golfo de California.

El consumo per cápita de productos cárnicos ha tenido un repunte importante en los últimos años: el de pollo registró un aumento de 28.3\% de 1997 a 2001; en el caso de la carne de res, el mismo indicador pasó de 15.5 a 16.5 kilogramos; y la carne de cerdo registró un aumento de $25.8 \%$ en el mismo periodo. Ese comportamiento puede estar explicado por el hecho de que ese tipo de productos cuentan con una mayor aceptación entre el pú- 


\section{Figura I}

Consumo per cápita de productos pesqueros en el mercado nacional y en Sonora de 1990 a $2002(\mathrm{~kg})$

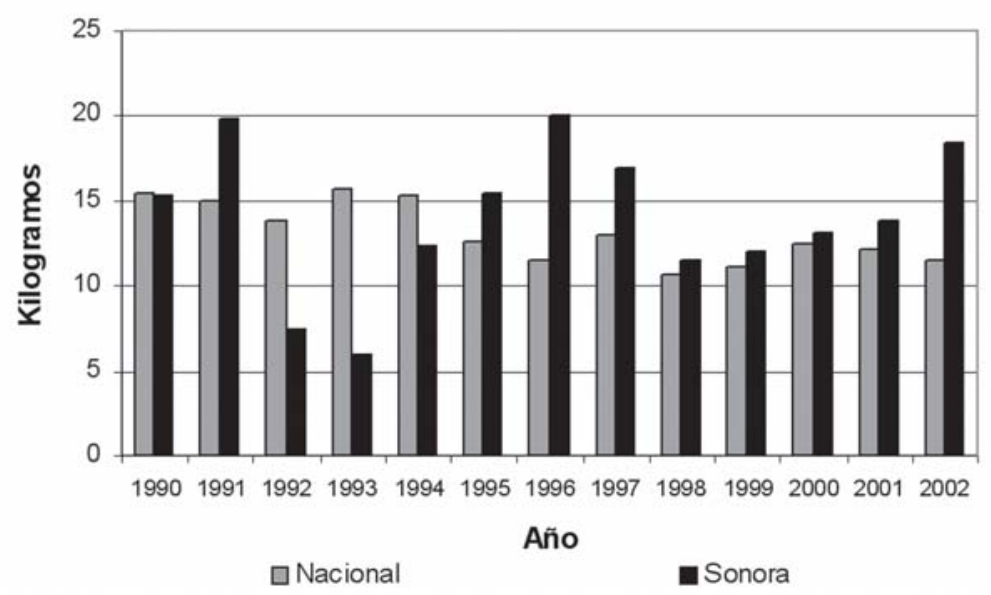

Fuente: Para consumo per cápita nacional, Conapesca (2001 y 2002). Para consumo per cápita de Sonora, Palafox (2003).

blico consumidor, pues constituyen un componente importante de la dieta de los mexicanos.

\subsection{Consumo per cápita de productos pesqueros para México y Sonora}

De acuerdo con los datos del Anuario estadístico de pesca (Conapesca, 2001 y 2002), el consumo per cápita de productos pesqueros en el país ha sido variable. En 1990, ese consumo era de 15.4 kilogramos por persona; y durante esa década, el indicador fue variable, tal como se muestra en la figura I. Cabe señalar que a partir de 1995 empezó la baja en el consumo per cápita de productos pesqueros, que llegó hasta 11.5 kilogramos por habitante en el año 2002. El comportamiento anterior se refiere al ámbito nacional, por lo que se debe tener especial atención ante este fenómeno por ser un "termómetro" de los gustos y preferencias de los consumidores, y de su percepción respecto al acceso a productos marinos.

El consumo per cápita de productos pesqueros en Sonora también mostró un comportamiento variable. Alcanzó niveles máximos en 1991 y 1996 , con 19.8 y 20 kilogramos, respectivamente; y de 1999 hasta 2002 mostró una tendencia creciente (figura 
I). Ese incremento está asociado con la recuperación de las principales pesquerías en Sonora, lo que motivó además la creación y fortalecimiento de la infraestructura pesquera en ese estado. Es importante señalar que en 1993 el consumo per cápita de productos pesqueros fue menor en comparación con otros años, debido a la caída de las capturas totales en Sonora, las cuales disminuyeron en 22.2\% (Palafox, 2003); esa situación limitó la oferta de productos pesqueros en el mercado regional e impactó de manera negativa el consumo de ese tipo de alimentos.

\subsection{Factores que influyen en el consumo de productos pesqueros en México}

El bajo consumo de productos pesqueros ${ }^{12}$ en nuestro país está relacionado con factores como los hábitos alimenticios, el poco o nulo conocimiento sobre las características nutricionales de los pescados y mariscos (motivado principalmente por la falta de promoción para el consumo de este tipo de alimentos), la estacionalidad en el consumo de productos de origen marino (que se concentra principalmente en época de cuaresma y fin de año), y el estrato socioeconómico. ${ }^{13}$

El consumo de pescados y mariscos en México está asociado con los niveles de ingreso de la población. Según datos de la Encuesta Nacional de Ingreso y Gasto de los Hogares (INEGI, 2000: 223-224), solamente $14.96 \%$ de las familias que se encuentran en el primer decil, el de los más pobres, incluyen en su dieta productos pesqueros, para lo cual emplean 1.04\% de sus ingresos. En lo que se refiere al último decil, el de la población con mayor poder de compra, $39.25 \%$ de los hogares incluyen en su

${ }^{12}$ El presidente del Consejo Mexicano de Promoción de Productos Pesqueros y Acuícolas (Comepesca) señaló que los productos pesqueros representan menos de 1\% de las ventas de autoservicio y $5 \%$ de los restaurantes. El consumo está concentrado en la época de fin de año y cuaresma, periodos en los cuales se genera $60 \%$ de las ventas (Castro, 2004).

${ }^{13}$ Respecto a los factores o variables que inciden en el consumo en general, Ferber (1973, citado en Villezca y Martínez, 2002: 357) señaló que la respuesta en el consumo de alimentos por parte de las familias ante los cambios en las variables económicas como ingreso y precio, tiene una importancia muy relevante en términos de política económica. Por su parte, Brown y Deaton (1972, citados en Villezca y Martínez, 2002: 357) señalaron que la composición de la familia, tanto por edad como por sexo, influye cualitativa y cuantitativamente en los patrones de consumo de alimentos. El nivel de educación del jefe de familia está relacionado con la forma como se distribuye el gasto en alimentos más nutritivos o que conduzcan hacia una dieta más balanceada. El estrato social al que pertenezca la familia determina los hábitos alimenticios. 
dieta productos de este sector, empleando en ello solamente $0.49 \%$ de su gasto. ${ }^{14}$

En el consumo de pescados y mariscos influye también la ubicación geográfica de la población. Se consume en mayor medida en las zonas urbanas, lo cual está relacionado con el hecho de que los principales mercados y centros de distribución se encuentran en las grandes ciudades. Los dos mercados más grandes e importantes en nuestro país, por el volumen y cantidad de productos de origen marino que manejan, son el mercado de la Nueva Viga, en la Ciudad de México, y el mercado de Zapopan, en Jalisco. Es importante destacar que esa concentración de productos pesqueros provenientes de todo el país genera altos precios para el consumidor final, porque da lugar a reembarques que, comercializados por ejemplo en el mercado de la Nueva Viga, tienen como destino su propio lugar de origen (Fernández, 1986: 352-353).

En México se captura un importante número de especies de pescados y mariscos; sin embargo, la mayoría se destinan al mercado externo, razón por la cual los consumidores nacionales conocen sólo los productos que se ofrecen en el mercado local, y que en ocasiones son de una menor calidad y presentación (es decir, en una presentación principalmente a granel, enhielado y sin datos nutricionales), situación que poco favorece su consumo. Es fundamental conocer el comportamiento del consumidor si se pretende fomentar el consumo de productos pesqueros dentro del país. Si bien es cierto que el mercado exterior es importante por las divisas que genera, también es importante reactivar el mercado nacional dado que estos productos brindan la oportunidad a la población nacional de bajos ingresos de una buena alimentación a precios accesibles.

${ }^{14}$ Daniel Vázquez y Pedro Villezca (2000: 99), en un estudio de estimación de la influencia de variables socioeconómicas sobre los gastos de consumo en pescados y mariscos para las familias de distintos estratos sociales del Área Metropolitana de Monterrey, señalan que de acuerdo con los resultados, entre más alto el estrato social, mayor es la proporción de familias que registró consumo de esos productos. La cantidad de dinero que se invierte en alimentos puede ser diferente, dependiendo del estrato económico. Mary Douglas y Baron Isherwood (1990: 113) señalan que los alimentos son la clase de mercancías en las que los pobres gastan la mayor parte de su ingreso. Esta proporción se mantiene de manera tan uniforme y tan vasta en el mundo entero, que es conocida como la ley de Engel. Los alimentos son también el artículo de primera necesidad por antonomasia. Por su parte, Moctezuma y otros autores (1989:174) mencionan que la proporción del gasto en alimento decrecerá respecto al gasto total conforme el ingreso se incremente. 
El caso del calamar gigante no es ajeno a lo que sucede con otros productos pesqueros en nuestro país, ya que su consumo es bajo y ha crecido lentamente, a pesar de su precio accesible y su alto contenido nutritivo. El calamar gigante es una especie marina que ha sido subutilizada en el mercado nacional: $89 \%$ se destina a la exportación, quedando sólo $11 \%$ en el mercado nacional, en donde se comercializa principalmente fresco, congelado y precocido (Salinas et al., 2003: 28).

El calamar gigante es un producto cuyo consumo puede aprovechar las características actuales de los consumidores. En los últimos años, el consumidor ha mostrado un mayor interés por cuidar su salud, lo que hace que se presenten interesantes oportunidades en estos segmentos. De igual forma, con la creciente integración de la mujer al mercado de trabajo, cada día se incrementa más el hábito de utilizar productos listos para consumirse y con una connotación de saludables. Según la Asociación Nacional de Tiendas de Autoservicio y Departamentales (ANTAD, citado en Benassini, 2001, Anexo 1), 90\% de los consumidores se preocupa mucho por la nutrición. Por otro lado, $48 \%$ de los compradores se preocupa por el contenido de vitaminas o minerales en sus alimentos; 19\% se preocupa por el contenido calórico, y solamente $16 \%$ tiene inquietud por el contenido graso. Estos son datos que deben considerarse al momento de desarrollar productos orientados al consumo humano directo.

Como se ha señalado, el calamar es un producto con importantes características nutricionales y que tiene un bajo precio; no obstante, el consumo nacional es bajo. Por ello se considera necesario promover el consumo del calamar gigante con programas específicos que difundan y promuevan sus características, con objeto de que este producto pueda tener una amplia aceptación entre el público consumidor. Además, debe proporcionarse orientación al consumidor, a los profesionales de la salud, y a toda persona que lo solicite en lo referente al calamar gigante. Es necesario también que se apoye la realización de estudios e investigaciones que permitan ampliar el conocimiento sobre este producto. Lo anterior es importante, ya que, como lo mencionan Cifuentes y otros autores (1997, cap. 1), cada día un número mayor de mexicanos tiene carencias de alimento, y se presenta la paradoja de una población mal nutrida en un país que cuenta con recursos naturales, específicamente pesqueros, de alto valor nutritivo. 


\section{Gustos y preferencias de consumo del calamar gigante en el mercado nacional y en Sonora}

El calamar gigante presenta bajos niveles de consumo per cápita en el mercado nacional (cuadro 4). Con el objetivo de identificar gustos y preferencias en el consumo del calamar gigante en el mercado nacional, se realizó un estudio de mercado de febrero a marzo de 2004 para determinar los factores que inciden en el consumo de ese producto pesquero. El estudio de mercado consideró como instrumento principal la aplicación de encuestas a consumidores de pescados y mariscos. El instrumento de muestreo utilizado fue diseñado de tal manera que el encuestado respondiera sobre su preferencia general en el consumo del calamar gigante.

Se aplicaron un total de 1,799 encuestas en 14 ciudades del país. El tamaño de la muestra de la población encuestada fue calculado para cada ciudad de acuerdo con el método planteado por William Cochran (1989), tomando en cuenta el último censo poblacional (INEGI, 2000). Se realizó un muestreo aleatorio sin reposición, considerando como población objetivo aquella que se encontraba en los principales centros comerciales, mercados municipales y centros de distribución de pescados y mariscos del país. El número de encuestas calculadas y aplicadas se muestra en el cuadro 5.

Todas las encuestas fueron realizadas durante el día en el intervalo de las 9:00 a las 15:00 horas. El muestreo se realizó entre el 15 de febrero y el 15 de marzo de 2004. Como parte de la metodología para la aplicación de las encuestas, se solicitó autorización de las principales cadenas comerciales, centros comerciales y mercados de las ciudades seleccionadas. Se solicitó la autorización correspondiente a las empresas de la Organización Soriana, s.A. de C.v., ubicada en la ciudad de Monterrey, Nuevo León; empresas Ley, ubicadas en Culiacán, Sinaloa; empresas del Centro Comercial Californiano, ubicado en La Paz, B.C.S.; mercados municipales de las diferentes ciudades y algunas pescaderías locales, así como a la Administración de los mercados de la Nueva Viga, en el Distrito Federal, y el Mercado del Mar, ubicado en Zapopan, Jalisco.

Los resultados de la encuesta revelan la situación actual del consumo del calamar gigante en el mercado nacional. Los principales resultados de la encuesta están orientados a describir elementos relacionados con el consumo del calamar gigante, la frecuencia y la cantidad en que se consume, además de las 
Cuadro 5

Ciudades encuestadas y número de encuestas levantadas

\begin{tabular}{lcccc}
\hline Cindad & Estado & $\begin{array}{l}\text { Población } \\
\text { objeto }\end{array}$ & $\begin{array}{c}\text { Encuestas } \\
\text { calculadas }\end{array}$ & $\begin{array}{c}\text { Encuestas } \\
\text { levantadas }\end{array}$ \\
\hline Ensenada & BC & 83,830 & 15 & 40 \\
La Paz & BCS & 66,451 & 12 & 45 \\
Guaymas & SON & 36,854 & 9 & 57 \\
Navojoa & SON & 33,294 & 6 & 59 \\
Cd. Obregón & SON & 94,148 & 17 & 60 \\
Hermosillo & SON & 210,488 & 38 & 69 \\
Culiacán & SIN & 202,070 & 36 & 60 \\
Los Mochis & SIN & 74,603 & 14 & 68 \\
Guadalajara & JAL & 686,477 & 225 & 156 \\
Cd. de México y & D.F.-EDO & & & 1044 \\
$\quad$ zona conurbada & MEX & 4 '462,361 & 813 & 101 \\
Monterrey & NL & 447,444 & 92 & 20 \\
Tuxtla Gutiérrez & CHI & 93,143 & 16 & 20 \\
Villahermosa & TAB & 88,082 & 15 & 1,799 \\
& & TOTAL & 1,308 & \\
\hline
\end{tabular}

Fuente: Elaborado a partir de las encuestas aplicadas a consumidores en el mercado nacional (2004).

\section{Cuadro 6}

Características socioeconómicas de la población encuestada por estado

\begin{tabular}{lccc}
\hline Estado & $\begin{array}{c}\text { Ingreso promedio } \\
\text { al mes (pesos) }\end{array}$ & $\begin{array}{c}\text { Numero de inte- } \\
\text { grantes promedio } \\
\text { porfamilia }\end{array}$ & Edad promedio \\
\hline Baja California & 5,500 & 4 & $36 \pm 11.9$ \\
Baja California Sur & 5,000 & 4 & $38 \pm 10.1$ \\
Chiapas & 4,900 & 4 & $37 \pm 10.7$ \\
D.F. & 10,000 & 4 & $39 \pm 8.1$ \\
Edo. de México & 9,000 & 4 & $38 \pm 9.9$ \\
Jalisco & 5,000 & 4 & $39 \pm 12.0$ \\
Nuevo León & 5,000 & 4 & $43 \pm 14.5$ \\
Sinaloa & 4,000 & 4 & $38 \pm 11.3$ \\
Sonora & 3,000 & 4 & $40 \pm 13.9$ \\
Tabasco & 5,500 & 4 & $41 \pm 12.1$ \\
\hline
\end{tabular}

Fuente: Elaborado a partir de las encuestas aplicadas a consumidores en el mercado nacional (2004).

presentaciones de ese producto que los encuestados estarían dispuestos a consumir en el mercado. Uno de los resultados más relevantes es que del total de la población encuestada que mencionó ser consumidor de pescados y mariscos, sólo 14.6\% consume calamar gigante. 
Es importante mencionar que $84.5 \%$ de los encuestados que manifestaron que sí consumen calamar gigante se encuentran en el rango de ingresos de entre 1,000 y 10,000 pesos mensuales, lo que indica que ese producto representa una alternativa de alimentación para ese grupo de la población.

De ese $84.5 \%$ que sí consume calamar gigante, $48.7 \%$ indicó que lo hace con una frecuencia de una a dos veces al mes, en tanto que $19.8 \%$ lo consume sólo eventualmente (en temporada o en cuaresma). En el caso de Sonora, el consumo de ese producto es más alto en comparación con el promedio nacional: $38 \%$ de los encuestados consume calamar gigante una vez por semana; $10 \%$ lo consume más de una vez, y 17\% lo consume dos veces al mes. El mayor consumo del calamar gigante en Sonora puede estar explicado por la ubicación geográfica de ese estado y por el fácil acceso que se tiene a los productos de origen marino.

Las encuestas aplicadas mostraron también resultados sobre la cantidad y la presentación del calamar gigante que compran los consumidores. Los resultados para el país indican que 67.1\% de los encuestados prefieren comprar calamar gigante fresco, en tanto que $32.9 \%$ prefieren comprarlo en presentaciones con valor agregado; por ejemplo, cocido. El alto porcentaje en la preferencia de compra del calamar gigante fresco se explica porque ésa es la presentación que más se encuentra en el mercado nacional, y porque así se acostumbra comprar los mariscos.

Ochenta y siete por ciento de los encuestados señaló que no preparan el calamar en casa debido a que no lo saben cocinar, ya que desconocen la forma de cocción del producto. Este resultado indica que existe una oportunidad de mercado para el calamar gigante con valor agregado.

En lo que se refiere a las cantidades en las que se compra ese producto, $47.6 \%$ de los encuestados compran entre uno y dos kilogramos, y 79.9\% de ese consumo se concentra en los hogares con entre tres y cinco integrantes de familia.

En el caso de Sonora, del total de los encuestados que sí consumen calamar, 50\% compra más de dos kilos; 30\%, de uno a dos kilos, y 20\%, menos de un kilo.

De acuerdo con los resultados de la encuesta aplicada, se determinó que el bajo consumo del calamar gigante en el ámbito nacional está relacionado con factores diversos. Uno de ellos tiene que ver con el limitado conocimiento que se tiene del calamar gigante: $23 \%$ de los encuestados señalaron que no lo consumen 
debido a que no lo conocen; sin embargo, 70\% manifestó que sí estarían dispuestos a consumirlo si conocieran el producto y su forma de preparación, lo cual plantea la necesidad de promoción. Otro factor está relacionado con el mínimo conocimiento sobre la forma de preparación del calamar gigante, particularmente del proceso de cocción: sólo $26 \%$ de los encuestados indicó que conoce alguna forma de preparación. Los gustos y las preferencias son factores que deben ser considerados: 20\% de los encuestados señalaron que no consumen calamar gigante debido a que no les gusta, en tanto que 19\% no lo consume porque no se les antoja, situación que puede estar relacionada con la presentación del producto en el mercado.

El estudio generó resultados sobre las presentaciones del calamar gigante que estarían dispuestos a consumir los encuestados (figura II). Para presentar esa información se elaboró un índice de importancia, que refleja los gustos y preferencias del consumidor en el país y en cada estado muestreado. Se calculó de la siguiente manera:

$$
\mathrm{IDI}=1 / \sum \frac{\mathrm{x}}{\mathrm{n}}
$$

\section{Figura II}

Índice de importancia de gustos y preferencias en el consumo del calamar gigante en México

\section{México}

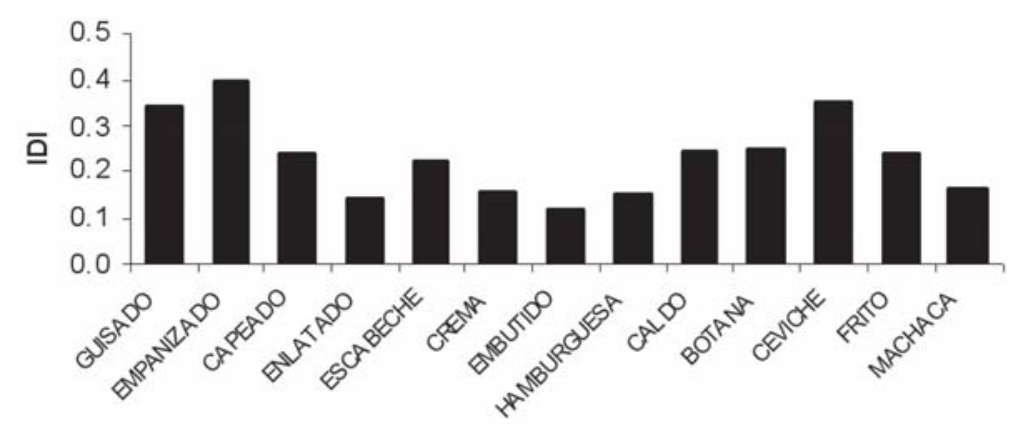

Fuente: Elaborado a partir de las encuestas aplicadas a consumidores en el mercado nacional (2004). 
Donde: $\mathrm{x}=$ valor otorgado por el encuestado para cada $\mathrm{X}$ tipo de preparación; $n=$ número de veces que aparece el tipo $\mathrm{X}$ de forma de cocinar el calamar.

Para visualizar de forma lógica los valores de este índice, se obtuvo su inverso, de tal manera que a mayor valor del índice de importancia (IDI), mayor es la preferencia de esa forma de preparación.

En términos generales, la población encuestada estaría dispuesta a consumir calamar gigante empanizado, en ceviche y guisado. Es importante destacar que el consumo en esas presentaciones tiene su base en la compra de calamar cocido. Esta situación resalta la necesidad de comercializar calamar gigante con valor agregado; por ejemplo, cocido, lo cual ofrece una oportunidad de mercado para el producto, ya que actualmente la principal presentación en la que se ofrece al consumidor final es fresco y con poco valor agregado.

\section{Conclusiones}

El calamar gigante representa una alternativa de alimentación para el consumidor nacional. Este producto posee características nutricionales que lo distingue de la carne de pollo, res y cerdo, y que lo ubican como un sustituto de esos alimentos, e incluso de otros alimentos de origen marino como el pulpo. El valor nutritivo del calamar, además del bajo precio al consumidor, representan una ventaja para este producto en el mercado.

Ante un escenario de bajo consumo del calamar gigante, se requieren esquemas de fomento que promuevan el producto. Es elemental, para ello, que el calamar gigante sea ofertado con valor agregado para que mejore su presentación y para que pueda figurar en los gustos y preferencias del consumidor, ello por medio de esquemas de comercialización que promuevan un aprovechamiento integral del recurso. Esto a su vez permitiría un beneficio para los pescadores que tienen como una de sus principales fuentes de ingreso la captura de ese producto.

El gobierno, por medio de las instancias correspondientes, puede participar en los esquemas de fomento y comercialización, lo que se podría concretar con el apoyo a los productores para que se organicen a fin de llevar a cabo actividades de comercialización conjunta y de posicionamiento en el mercado. Ante ese escenario se estaría en posibilidades de fortalecer la estructura productiva de esa pesquería, repercutiendo en mayores beneficios para los productores primarios; es decir, los pescado- 
res, y para el consumidor final, con la oportunidad de ofrecerles un producto económicamente accesible y de alto valor nutritivo.

\section{Bibliografía}

Benassini, Marcela (2001), Introducción a la investigación de mercados, Pearson Educación, México.

Cochran Gemmell, William (1989), Sampling Techniques, Willey and Sons, Inc., Nueva York.

Conapesca (Comisión Nacional de Acuacultura y Pesca) (2000), Anuario estadístico de pesca, Conapesca, México.

(2001), Anuario estadístico de pesca, Conapesca, México. (2002), Anuario estadístico de pesca, Conapesca, México. (2003), Anuario estadístico de Pesca, Conapesca, México.

Douglas, Mary y Baron Isherwood (1990), El mundo de los bienes: hacia una antropología del consumo, Editorial Grijalbo, s.A., colección Los Noventa, México.

Ezquerra Brauer, Josafat Marina, Norman Haard F., Rosalina Ramírez Olivas, Hermenegildo Olivas Burrola y Carlos J. Velásquez Sánchez (2002), "Influence of Harvest Season on the Proteolytic Activity of Hepatopancreas and Mantle Tissues from Jumbo Squid (Dosidicus gigas)", Journal of Food Biochemistry, Connecticut, 26: 459-475.

FaO-OMS-UnU (Organización de las Naciones Unidas para la Agricultura y la Alimentación-Organización Mundial de la Salud-Universidad de las Naciones Unidas) (1985), $\mathrm{Ne}$ cesidades de energía y de proteinas. Informe de una Reunión Consultiva Conjunta FAO/OMS/UNU de Expertos, Serie de Informes Técnicos, núm. 724, Ginebra.

Fernández de la Masa, Guillermo (1986), "La comercialización y el abasto de productos pesqueros. Análisis y perspectivas", en Desarrollo pesquero mexicano 1985-1986, tomo II, Secretaría de Pesca, México, pp. 346-361. 
Klett Traulsen, Alexander (1996), "Pesquería del calamar gigante Dosidicus gigas”, en M. Casas y G. Ponce (eds.), Estudio del potencial pesquero y acuícola de Baja California Sur, vol. I, CIB-CICIMAR-UABCS, México, pp. 127-149.

Markaida, Don Unai (2001), Biología del calamar gigante Dosidicus gigas Orbigny, 1835 (Cephalopoda: Ommastrephidae) en el Golfo de California, México, tesis para cubrir parcialmente los requisitos necesarios para obtener el grado de doctor en Ciencias, Centro de Investigación Científica y de Educación Superior de Ensenada, Ensenada, Baja California, México.

Moctezuma Hernández, Patricia (1989), “Canales de comercialización y de formación de precios de los productos pesqueros”, en M. Siri y P. Moctezuma (eds.), La pesca en Baja California Sur, Universidad Autónoma de Baja California, Mexicali, B.C., México, pp. 145-164.

, Alejandro Mungaray Lagarda y Jesús Robles Villa (1989), "Estructura del consumo regional de productos pesqueros”, en M. Siri y P. Moctezuma (eds.), La pesca en Baja California Sur, Universidad Autónoma de Baja California, Mexicali, B.C., México, pp. 165-183.

Palafox Moyers, Carlos Germán (2003), Análisis socioeconómico de la pesca y la acuacultura en Sonora 1990-2002, Universidad de Sonora, Sonora, México.

Pindyck, Robert y Daniel Rubinfeld (2001), Microeconomía, $5^{\text {a }}$ ed., Prentice Hall, Madrid.

Presidencia de la República, Poder Ejecutivo Federal (2003), Tercer Informe de Gobierno, Anexo estadístico, México, septiembre.

Rivera Parra, Iván (2001), La pesquería del calamar gigante Dosidicus gigas (d'Orbigny, 1835) en el Golfo de California, tesis doctoral, Universidad de Colima, México.

Salinas Zavala, Cesar Augusto, Susana Camarillo Coop, Arminda Mejía Rebollo, Saúl Sánchez Hernández, Carolina Sán- 
chez Verdugo, Marcelo González Angulo, Edwin Flores Quintana y Luis Felipe Beltrán Morales (2003), Alternativas para fortalecer la cadena productiva de la pesquería de calamar gigante, Centro de Investigaciones Biológicas del Noroeste, La Paz, B.C.S., México.

Coop, Arminda Mejía Rebollo, Saúl Sánchez Hernández, Carolina Sánchez Verdugo, María Consepción Luna Raya, Francisco Javier de la Cruz González, Alberto Aragón Noriega, Gastón Bazzino Ferreri y Luis Felipe Beltrán Morales (2004), Cadena productiva del calamar gigante, segunda etapa: aseguramiento de la materia prima y determinación de preferencias de consumo en México y en el mundo, Centro de Investigaciones Biológicas del Noroeste, La Paz, B.C.S., México.

Valencia Aramburo, Moisés (1988) Economía pesquera, Universidad Autónoma de Sinaloa, Sinaloa, México.

Vázquez Cotera, Daniel y Pedro A. Villezca Becerra (2000), “Forma funcional y modelos de respuesta censurada en el análisis de consumo de atún, de pescados y mariscos en los hogares del área metropolitana de Monterrey, Ensayos, Universidad Autónoma de Nuevo León, Nuevo León, México, noviembre, xIX (2): 85-120.

Villezca Becerra, Pedro e Irma Martínez Jasso (2002), “Efectos de factores socioeconómicos en el consumo de alimentos en el Área Metropolitana de Monterrey", Ciencia UANL, Nuevo León, México, julio-septiembre, v (3): 367-357.

Zugarramurdi Aurora y María A. Parín (1998), Ingeniería económica aplicada a la industria pesquera, Documento Técnico de Pesca 351, FAO, Roma-Italia.

\section{Hemerografía}

Cordova Murueta, Julio Humberto (2005), "Potencian nutrientes del calamar gigante”, Reforma, sección Ciencia, 25 de agosto. 
Recursos electrónicos

Castro, Raúl (2004), “Tienen empresas pesqueras gran potencial para vender a Japón”, Milenio, 27 de septiembre.

Cifuentes Lemus, Juan Luis, Pilar Torres García y Marcela Frías M. (1995), "El océano Ix. La pesca”, en M. C. Farías (coord.), La ciencia para todos, $2^{\text {a }}$ ed., Fondo de Cultura Económica, México. http://omega.ilce.edu.mx:3000/sites/ ciencia/volumen $2 /$ ciencia3/081/htm/oceano.htm, 12 de octubre 2004.

(1997), "El océano y sus recursos x. Pesquerías", en M. A. Pulido y M. C. Farías (coords.), La ciencia para todos, $2^{a}$ ed., Fondo de Cultura Económica, México, http:// biblioteca.redescolar.ilce.edu.mx/sites/ciencia/volumen2/ ciencia3/087/htm/oceano10.htm, 12 de octubre de 2004.

FAO-Latinfoods (Organización de las Naciones Unidas para la Agricultura y la Alimentación-Oficina Regional de América Latina y el Caribe) (2002), Tabla de composición de alimentos de América Latina, http://www.rlc.fao.org/bases/alimento/, 8 de noviembre 2004, (actualización: 24 de agosto de 2005).

http://www.caaarem.com.mx/COM/SPRENSA.NSF/0/67cc 6f8657f88586256f1c005d9b37?OpenDocument, $12 \mathrm{de}$ octubre de 2004.

INEGI (Instituto Nacional de Estadística, Geografía e Informática) (2000), Encuesta Nacional de Ingreso y Gasto de los Hogares, http://www.inegi.gob.mx/prod_serv/contenidos/espanol/bvinegi/productos/encuestas/hogares/enigh/2000/ enigh.pdf, 19 de noviembre de 2004.

Martínez Jasso, Irma (2001), "La disponibilidad de alimentos en México. Las hojas de balance alimentario de la FAO", Entorno Económico, Centro de Investigaciones Económicas, Universidad Autónoma de Nuevo León, Nuevo León, México, mayo-junio, Xxxix (232): 11-19, http://www.uanl. $\mathrm{mx} / \mathrm{facs} / \mathrm{fe} /$ publicaciones/entorno/may-jun01.pdf, $20 \mathrm{de}$ noviembre de 2004. 
ONU-CEPAL (Organización de las Naciones Unidas-Comisión Económica para América Latina y el Caribe) (1991), Determinación de las necesidades de energía y proteinas para la población de diez países latinoamericanos, http://www. paho.org/Spanish/dpm/shd/hp/hapl04_cepal_reqnut.pdf, 31 de agosto de 2005 .

Profeco (Procuraduría Federal del Consumidor) (2004), Quién es quién en los precios de carne de pollo, cerdo, res, pescados y mariscos, http://www.profeco.gob.mx/html/precios/ quienesquien.htm, 9 de noviembre de 2004.

Sagarpa (Secretaría de Agricultura, Ganadería, Desarrollo Rural, Pesca y Alimentación), Dirección General de Ganadería (2002), Consumo aparente y per cápita de carnes, http:// sagarpa.gob.mx/Dggl>, 20 de febrero de 2002 (actualización: 26 de febrero de 2002).

Ma. Consepción Luna Raya. Licenciada en economía agrícola por la Universidad Autónoma Agraria Antonio Narro (UAAAN), Saltillo, Coahuila, y maestra en ciencias en administración integral del ambiente por El Colegio de la Frontera Norte (Colef), Tijuana, B.C. Actualmente es estudiante de doctorado en el Centro de Investigaciones Biológicas del Noroeste (Cibnor, s.C.), La Paz, B.C.s. Es miembro del Proyecto Binacional para Asuntos Ambientales entre el Colef y la Compton Foundation, Inc. (20012002), y miembro de la Sociedad Mexicana de Pesquerías desde mayo de 2005. Es consultora de tiempo parcial de wwF-México Programa Golfo de California, y colaboradora en el proyecto "Cadena productiva de la pesquería de calamar gigante, segunda etapa", realizado por el Cibnor y financiado y publicado por Conapesca (2004).

José Isabel Urciaga García. Licenciado en economía por la Universidad Autónoma de Nayarit, México, y doctor en ciencias económicas y empresariales por la Universidad Autónoma de Barcelona, España. Actualmente es profesor-investigador en la Universidad Autónoma de Baja California Sur (UABCS), y es miembro del Sistema Nacional de Investigadores (SNI), académico nacional, de la Academia de Ciencias Económicas, A.C., de la Sociedad Internacional de Ciencias Regionales, y de la Red Internacional 
de Investigadores en Ciencias Sociales y Humanidades. Sus líneas actuales de investigación son: desarrollo y sostenibilidad, desarrollo territorial, regional y local, economía y política de los recursos naturales y ambientales, economía y política del turismo, análisis del mercado de trabajo, y econometría aplicada. Publicaciones recientes: "Los rendimientos privados de la escolaridad formal en México", Comercio Exterior, vol. 52, núm. 4, abril de 2002, pp. 324-330; "Las remesas. Una opción estratégica para el financiamiento de desarrollo local", en Jerjes Aguirre y Oscar Pedraza, Migración internacional y remesas en México, Universidad Michoacana de San Nicolás de Hidalgo, Michoacán, México, 2004, pp. 101-114; "Turismo alternativo. Una estrategia de desarrollo del espacio rural para Baja California Sur”, Prospectiva Económica, Universidad Michoacana de San Nicolás de Hidalgo, núm. 5, julio diciembre de 2004, pp. 177-197, y con Abelino Torres, "Algunos aspectos importantes sobre la viabilidad de los distritos industriales en la economía mexicana”, Expresión Económica, Facultad de Economía, Universidad de Guadalajara, núm. 10, noviembre de 2003, pp. 12-32.

Cesar Augusto Salinas Zavala. Doctor en ecología marina por el Centro de Investigación Científica y de Educación Superior de Ensenada, Ensenada, B.C; investigador del Centro de Investigaciones Biológicas del Noroeste (Cibnor, s.c.), y miembro del Sistema Nacional de Investigadores (SNI). Sus líneas actuales de investigación son: ecología marina y pesquerías. Publicaciones recientes: "Sobre la respuesta al cambio climático en el noroeste de México”, CIENCIA, vol. 51, núm. 3, 2000, pp. 11-18; con R. Morales-Azpeitia y J. López-Martínez, "La pesca de calamar gigante (Dosidicus gigas) en el Golfo de California”, JAINA, Boletín informativo EPOMEX, vol. 8, núm. 1, 1997, pp. 6-7; con L. Brito-Castillo, E. Alcántara-Razo y R. Morales-Azpeitia, "Water Temperatures in the Gulf of California in May and June 1996 and their Relation to the Capture of Giant Squid (Dosidicus gigas, D; Orbigny, 1835)”, Ciencias Marinas, vol. 26, núm. 3, 2000, pp. 413-440, y con A. V. Douglas y H. F. Diaz, "Inter-annual Variability of NDVI in Northwest Mexico. Associated Climatic Mechanisms and Ecological Implications", Remote Sensing and Environment, vol. 82, núms. 2-3, 2001, pp. 417-430.

Miguel Ángel Cisneros Mata. Doctor en ciencias por la Universidad de California. Actualmente es coordinador eco-regional del 
Programa Golfo de California del Fondo Mundial para la Naturaleza (WWF-México). Sus líneas de investigación son: formulación de modelos biológico-pesqueros, desarrollo y conservación. Publicaciones recientes: con M. O. Nevárez-Martínez, A. Hernández-Herrera, E. Morales-Bojórquez, A. Balmori-Ramírez y R. Morales-Azpeitia, "Biomass and Distribution of the Jumbo Squid (Dosidicus gigas; d'Orbigny, 1835) in the Gulf of California, Mexico", Fisheries Research, núm. 49, 2000, pp. 129-140, y con E. Morales-Bojorquez, Manuel O. Nevares-Martínez y Agustín Hernández-Herrera, "Review of Assessment and Fishery Biology of Dosidicus gigas in the Gulf of California, Mexico", Fisheries Research, núm. 54, 2001, pp. 83-94.

Luis Felipe Beltrán Morales. Licenciado en economía por la Universidad Autónoma de Baja California Sur (UABCS), y doctor en ciencias ambientales por el Centro EULA de la Universidad de Concepción, Chile. Actualmente es investigador y coordinador de Estudios Ambientales del Centro de Investigaciones Biológicas del Noroeste (Cibnor, s.c.), miembro del Sistema Nacional de Investigadores (SNI) y de la Asociación Latinoamericana, profesor de la Maestría en Economía del Medio Ambiente y Recursos Naturales de la UABCS, y delegado de Medio Ambiente ante el California Border Environmental Cooperation Committee (cal/ BECC) y la Comisión de las Californias (Comcal). Publicaciones recientes: "Consumo sustentable como derecho-obligación para disfrutar de un medio ambiente sano", Región y Sociedad, vol. 14, núm. 23, 2002, pp. 193-198; con F. García-Rodríguez, J. Borges Contreras, G. Sánchez-Mota y A. Ortega Rubio, "Environmental and Socioeconomic Multivariate Analysis of the Primary Economic Sector of Mexico", Sustainable Development, vol. 11, núm. 2, 2003, pp. 77-83; con H. A. González O., C. Cáceres-Martínez, H. Ramírez, S. Hernández-Vázquez, E. Troyo-Diéguez y A. Ortega-Rubio, "Sustainability Development Analysis of Semi-intensive Shrimp Farms in Sonora, Mexico", Sustainable Development, vol. 11, núm. 4, 2003, pp. 213-222; con F. García-Rodríguez, J. Borges-Contreras y A. Ortega-Rubio, "Apertura comercial y medio ambiente", Interciencia, vol. 27, núm. 5, 2002, pp. 259-263, y con A. Ortega-Rubio, M. Lagunas, H. González, M. Soto y M. Acevedo, "Environmental, Economic and Social Effects caused by NAFTA in the Fishery Food Companies of Baja California Sur, Mexico", Sustainable Development, vol. 10, núm. 4, 2002, pp. 215-217. 